\title{
An Attentional View of Right Hemisphere Dysfunction
}

John A. (Tony) Seikel

Idaho State University, Pocatello, United States

Right hemisphere dysfunction (RHD) arises from lesions to the right hemisphere. For people with a dominant left hemisphere, the form and content of language appear normal until deep testing is performed. People with RHD have a high likelihood of demonstrating left hemispatial neglect, including left visual neglect. They will also likely show narrative deficits arising from failure to consider the needs of the listener, as well as reduced affect in speech and facies arising from deficient processing of suprasegmental details and facial manifestations of emotion. While these deficits seem disparate and disconnected, examination of the lesion site and its relationship to attention deficit underscores the powerful contribution of attentional failure to RHD. Further, it is possible that deactivation of the non-dominant hemisphere results in reduced manifestation of the right hemisphere regions associated with empathy, compassion, and integrated sense of self. The purpose of this article is to provide an overview of RHD and its underpinnings, and to discuss clinical implications of RHD.

Keywords: Right hemisphere dysfunction, Right hemisphere, Hemispatial neglect, Left neglect, Emotion, Attention, Empathy, Compassion, Speech

\section{INTRODUCTION}

As speech-language pathologists we are familiar with the notion of left hemisphere damage and its tremendous impact on language and speech function. We are all conversant in the results of injury to the dominant hemisphere, including the numerous aphasias, agraphia, alexia, and so forth. This paper is an effort to describe "the other half." RHD is something of the "silent partner" of left hemisphere dysfunction, but in many ways the result of right hemisphere lesion may be even more devastating to the individual than presence of aphasia. To this point, many of the deficits found in RHD may be attributed to a deficit of attention.

Expressive language presents as intact syntax, morphology, semantics and phonology, and all of these are largely within the domain of the dominant hemisphere [1-3]. An individual with RHD will likely have intact fine-motor control, will often show an absence of dysarthria or apraxia, and will have speech and language that appear quite normal on first investigation. The reason RHD is a "hidden" disorder is that it houses a striking constellation of problems, including a semantic deficit that appears to be rooted in a concreteness and that is not apparent upon casual observation [4]. There is likely a significant attentional element to the disorder, with a concomitant deficit in narrative language. Perhaps the most devastating component for both client and clinician is the client's lack of awareness of the problem, because this is a significant barrier to clinical success [5].

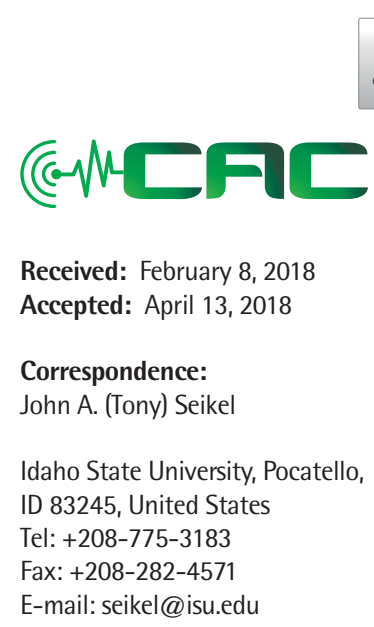

(C) 2018 The Korean Association of SpeechLanguage Pathologists

This is an Open Access article distributed under the terms of the Creative Commons Attribution NonCommercial License (http://creativecommons.org/ licenses/by-nc/4.0/) which permits unrestricted noncommercial use, distribution, and reproduction in any medium, provided the original work is properly cited. 
Before beginning this discussion it is important to make a distinction and a clarification. We refer to the left hemisphere as the dominant hemisphere, and the right hemisphere as the non-dominant hemisphere. Dominant hemisphere is shorthand for dominant for language, which, for between $87 \%$ and $89 \%$ of the population is the left hemisphere [6]. Sensation from the left half of the body is delivered to the right hemisphere, and sensation from the right half of the body goes to the left hemisphere. Motor organization follows the same decussation pattern. The non-dominant hemisphere, then, is typically the right hemisphere. For the other $11-13 \%$ of the population who are left-handed the dominant hemisphere is often the right hemisphere. For a subset of these individuals, there may be mixed laterality, where language and other "typically" left-hemisphere functions are distributed between the two hemispheres. (It should be noted that Knecht et al. [7] found that about $7 \%$ of right handed people have right hemisphere dominance for language.) The caveat for this paper is that I will use the term "left hemisphere" as a proxy for "dominant hemisphere," and "right hemisphere" to represent "nondominant hemisphere."

The goal of the present paper is to discuss the normal substrate, neurophysiology of attention and linguistic function of the right hemisphere, particularly related to differences between the left and right hemispheres. Following this will be a discussion of deficits found in RHD, particularly those of language (semantics and pragmatics), cognition, and attention, and specifically those related to hemispatial neglect and misidentification syndromes. Woven into this discussion are the personal and social impacts of RHD on the lives of the individuals and their families.

\section{Normal substrate}

It is important to distinguish left- and right-hemisphere function. While it is tempting to categorically identify specific locations to functions, the various regions of the brain actually operate within coordinated, distributed interactive systems [8]. The localizationist view of segregated functions for segregated structures has given way to a connectionist representation consisting of networks performing functions [9]. The reality of cortical function is that both hemispheres work together in intricate networks of interaction [10], and the complexity of this interaction is perhaps the strongest differentiator between cortical activity of humans and non-human primates [11]. That having been said, the neural networks required for complex cognitive and linguistic activities have developed in the context of specialized hemispheric centers.

The left hemisphere is dominant for the form of language, including syntax, morphology, and phonology [12] (see Table 1). Wernicke's area in the left temporal lobe is essential for receptive language function and comprehension. The left hemisphere is very detail oriented, while the right hemisphere places detail in context. The left hemisphere is a very rapid serial processor, moving information quickly and sequentially.

Table 1. Differences between left and right hemisphere function

Left hemisphere functions

Dominant for the structure of language, including syntax, morphology, and phonology

Dominant for language reception (Wernicke's area) and expression (Broca's area)

Processing of detail

Processing serial information

Judgment

Stimulus discrimination and categorization

Left dorsolateral prefrontal cortex (DLPFC) is prone to impulsivity and risktaking and distressed by pain

Responsible for analysis

Left fusiform gyrus identifies facial details

Left insula is implicated in motor planning

Left frontal lobe is critical for memory and recall of memory

Right hemisphere functions

May provide linguistic context

Is parallel processor of information

Appears to provide the gestalt of information

Places detail in context

Has slower responses than left hemisphere

Processes longer phonetic units (vowels)

Right DLPFC is reflective and relatively impervious to pain

Is responsible for synthesis

Right fusiform gyrus is responsible for facial recognition

Right insular cortex is location for our sentient nature, insight, awareness, compassion, empathy

Right operculum is site of secondary sensory cortex

Right inferior frontal cortex inhibits response of the left DLPFC

Has attentional networks

Is responsible for suprasegmental comprehension and expression

Is responsible for affect interpretation and expression

Is responsible for interpreting facial expression to determine affect

Comprehends and contextualizes humor, grief and joy 
The right hemisphere functions as a parallel processor, looking at all information at the same time. The right hemisphere appears to deal with the gestalt of information rather than individual details. The left hemisphere is extremely judgmental, capable of discerning minute differences between stimuli. This judgment component allows the left hemisphere to organize data based on categories, and the right hemisphere can view the data as a whole to provide context for those categories. The left hemisphere is a rapid processor and deals with the small units of speech (consonants), while the right hemisphere is slower and more ponderous $[13,14]$, processing longer phonetic units (vowels). The left hemisphere is impulsive and prone to risk-taking, while the right hemisphere is reflective [15]. The left hemisphere is responsible for analysis, while the right hemisphere is responsible for synthesis. The significant pathology of left hemisphere stroke is the language deficit of aphasia, while RHD is highlighted by hemispatial neglect.

There are marked physiological differences between the two hemispheres, particularly in the dorsolateral prefrontal cortex (DLPFC), temporoparietal juncture (TPJ), and insular cortex. Below is a discussion of these areas in detail. (Please see Figure 1 for Brodmann locations mentioned in the discussion).

\section{Dorsolateral prefrontal cortex}

The left DLPFC (Brodmann areas [BA] 9, 46) is extremely important for executive activity. Executive functions manipulate the basic cognitive elements of memory, perception, attention, visuospatial processes and linguistic processes in order to accomplish cognitive goals, make motor or cognitive plans, and execute complex tasks. Even the simplest process, such as warming a cup of coffee in the microwave, would be impossi- ble without the DLPFC. This region governs how we access memory and match it with intention to have our goals met. The left DLPFC has very rapid and impulsive processing, capable of snap decisions [15]. It is critical for memory and recall of memory [16].

In contrast, the right DLPFC is very reflective. It is a slower processor, and doesn't make rapid, impulsive decisions. It is relatively impervious to pain, while the left DLPFC appears to be quite easily distressed by it [17]. Most importantly for the present discussion, the right DLPFC is a major component of the attentional network [18].

\section{Fusiform gyrus}

The left fusiform gyrus (BA 37) is responsible for identifying facial details [19]. In contrast, the right fusiform gyrus is responsible for putting that information together. The left fusiform gyrus has a strong role in word identification, and is part of the ventral visual and auditory stream [20-22]. Together the left and right fusiform gyri perform the important function of face identification by integrating these functions: the left fusiform gyrus uses its exquisite capacity for feature identification, and the right hemisphere places those features into a seamless whole for the purpose of face recognition.

\section{Cuneate gyrus and precuneus}

The cuneate gyrus (BA 17) appears to have a role in self efficacy. The precuneus is involved in knowledge of self, while the cuneate gyrus is involved in perception of body.

\section{Insular cortex}

The insular cortex (BA 13) reveals a striking hemispheric dif-
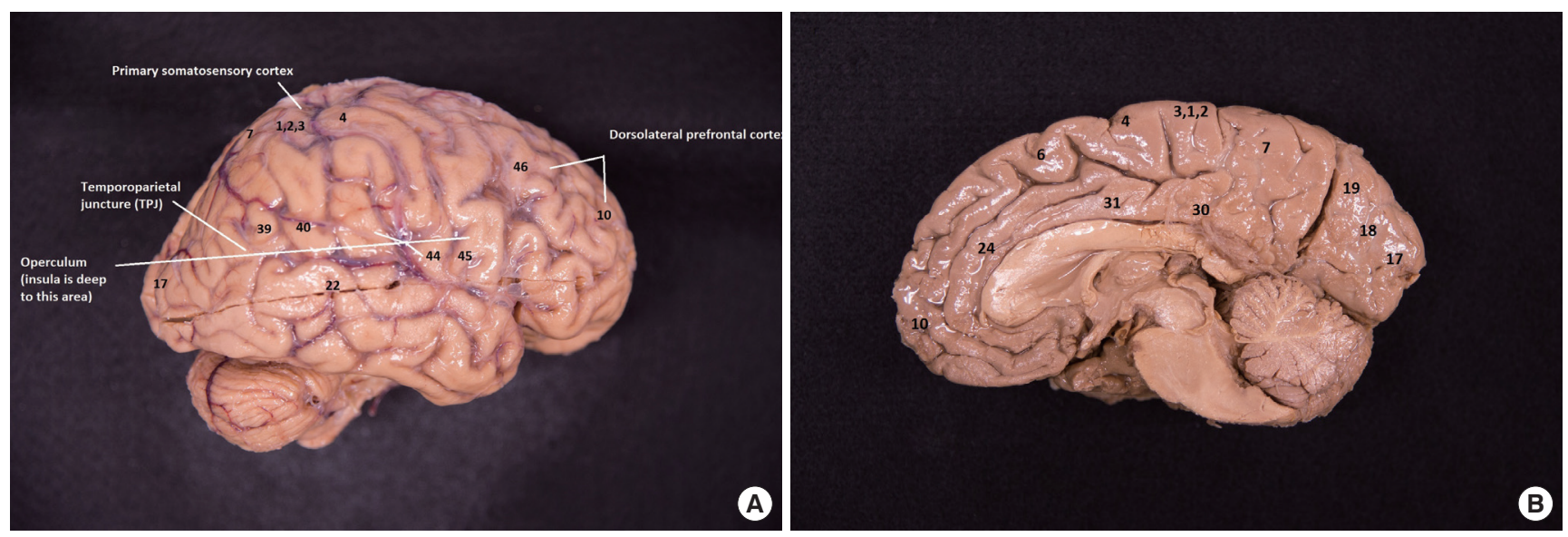

Figure 1. (A) Lateral view of cerebral cortex, with important regions noted. Note that numbers indicate Brodmann areas. (B) Medial view of cerebral cortex. 
ferentiation. The primal role of the insula is gustation but Dronkers [23] found that, in addition, the left insular cortex is critically involved in expressive language production, with a lesion to the insula causing apraxia of speech. While this finding was supported by Shuren [24], and Donnan et al. [25], and Nestor et al. [26], it has been questioned by other authors $[27,28]$. Both Hillis et al. [27] and Moser et al. [28] have identified non-insular lesion sites (e.g., left-hemisphere Rolandic operculum or motor cortex) for acquired apraxia, as well as individuals with focal left insular lesion who did not have apraxia of speech, sharply contradicting Dronkers. In all cases, the lesions were in the left hemisphere. The left insular cortex is embedded beneath the operculum of the left hemisphere, specifically beneath Broca's area (BA 44, 45). Broca's area of the left hemisphere is the center for expressive language, and a lesion to this area and the cortical white matter beneath it results in non-fluent aphasia. Thus, lesions to the left opercular region and the region immediately beneath it result in the constellation of speech and language deficits seen frequently in frontal lobe lesions, specifically non-fluent aphasia and apraxia of speech.

In contrast, the right insular cortex is not involved in speech or language at all, but rather is the seat of awareness [29]. The right insular cortex appears to be the location for our sentient nature, and Craig and Craig [29] refers to it as the "sentient cortex," because it is the location of insight, awareness, compassion, empathy, and that feeling we recognize as the "aha" moment. Although the primary sensory cortex is embodied in the primary sensory region (SI) of the postcentral gyrus (BA 1, $2,3)$, the insula and the overlying operculum are also the site of the secondary sensory cortex (SII). While sensation from the ventral surface of the body is projected onto SI of the cortex, sensation from the dorsum of the body (back of trunk, head, legs) is relayed on the right insular cortex and the opercular surface [30]. It is hypothesized that this supports social activities such as grooming for chimps or back massages for humans: The trust that is required for allowing someone to touch your body, out of sight, may well form a deep social bond. If this hypothesis holds, then the "trusting" act of allowing close social contact may well be a basis for empathy and compassion [31].

\section{Inferior frontal cortex}

The left inferior frontal cortex (specifically Broca's area, BA 44,45 ) is involved in semantic processing and is clearly implicated in aphasia. In sharp contrast, the right inferior frontal cortex is actively involved in inhibiting the response of the left DLPFC [32,33].

\section{Cingulate gyrus}

The cingulate gyrus is divided into anterior (ACC: BA 24,25) and posterior cingulate cortex (PCC: BA 29, 30, 23, and 31). The ACC is heavily involved in emotion and response regulation, as well as impulse control, empathy and cognition [35-37]. It is involved in motor control [38], and mediates oxytocin release in maternal-infant interaction [39]. The PCC is involved in concentration and focused attention, as well as evaluation of stimuli [38]. The cingulate gyrus is a critical link between the limbic system and the frontal cortex, seeming to allow moderation of limbic responses through cognitive activity, including biographical memory [40]. Significantly, loss of cingulate gyrus function can result in uninhibited activity of the limbic system [41].

\section{Neurophysiology of attention}

A central problem in RHD is attention. There are three basic attentional networks: Alerting attention, orienting attention, and executive attention [18]. The right hemisphere dominates all three forms of attention, and attention forms a bedrock deficit in RHD.

\section{Alerting attention}

The alerting network is the lowest level system, in the sense that it is an intrinsic element of physiological arousal. Its responsibility is to alert the cortex to the presence of a stimulus, and when a stimulus enters one's perception this network is activated. In people with traumatic brain injury (TBI), the clinician will often see this as the earliest network to re-emerge after coma [42], and its function can activate the limbic fear response that is unmodulated by frontal cortical input. Thus, the agitation seen in early consciousness may likely arises from uninhibited arousal of this network and the limbic system [43]. The alerting network is the domain of the right frontal, parietal, DLPFC, as well as the anterior cingulate cortex (ACC). It includes activation of the thalamus, signaling presence of a stimulus.

\section{Orienting attention}

The second of these networks is the orienting network. This network is responsible for identifying specific information from multiple stimuli [44]. When a person is alerted to the presence of a stimulus (danger or delightful), the orienting network allows the individual to differentiate it from other 
stimuli. There appears to be both exogenous and endogenous orienting. Exogenous orienting is orientation to an external stimulus, such as a sound or visual input. Endogenous orienting involves orientation based on some cognitive category or set [44]. Raz and Buhle [18] note that the orienting network involves the pulvinar (specifically the language nucleus of the thalamus), the superior colliculus, parietal lobe, temporal lobe, and frontal eye fields. Likely, the ventral visual stream (or "what" stream [45]) is activated by the orienting network, allowing one to identify the nature of a visual stimulus.

\section{Executive attentional network}

This is a very important network for executive function for the discussion of RHD. The functions attributed to this network are reflected in some the names associated with it: executive network, supervisory network, conflict resolution or focused attention network [18]. There are two important features of this network. First, it resides solely in the right hemisphere. Second, it requires a great deal of effort and energy to maintain it. It is not the default mode of operation, but rather requires effort by the individual to activate it.

There are two essential anatomical components to the executive attentional network: the TPJ and the right DLPFC. The TPJ resides at the juncture of the temporal and parietal lobes, near the angular gyrus (BA 39) and the superior temporal gyrus. The right and left DLPFC work together to control cognitive executive functions. The right DLPFC has the important role of focusing attention, on demand. At this juncture it is beneficial to recall that the right hemisphere region that corresponds to Broca's area on the left hemisphere is responsible for inhibiting the impulsive activity of the left DLPFC. Thus, the executive attention calls on the right DLPFC, right TPJ, and right inferior frontal lobe. Damage to any of these areas can result in attention deficit.

\section{Right hemisphere linguistic function}

There are four major areas of communication function of the right hemisphere: communication gestalt, suprasegmental production and perception and facial nuance, affect production and interpretation, and pragmatics.

\section{Suprasegmental aspects of speech}

The right hemisphere has responsibility for expression and interpretation of suprasegmental aspects of language and speech, including fundamental frequency and intensity modulation $[46,47]$.

\section{Affect interpretation and production}

Processing of affective information appears to be the domain of the right hemisphere. For example, as the right hemisphere interprets sad stimuli the left hemisphere remains markedly quiescent [48]. This differential processing may arise in part from the right hemisphere's capacity for interpreting nuance of vocal intonation [47] and facial expression for the purpose of identifying affect. The right hemisphere comprehends and contextualizes humor, grief and joy, most likely as a result of activation of the right insular cortex [49].

\section{Pragmatics}

A critical role of the right hemisphere is in pragmatics of communication. The right hemisphere utilizes its strength in Theory of Mind to "know" what the other person is thinking, and to actually care about the outcome of an interaction. An appropriate pragmatic communication includes sensitivity to the needs of others, which may require inhibiting responses that might seem otherwise appropriate [50], a function of the inferior frontal lobe of the right hemisphere (BA 44, 45). The knowledge of communicative context, suprasegmental aspects of speech, and communication gestalt allows the communicator the ability to comprehend nuance of communication, be sensitive to the needs of others, and to respond appropriately.

In summary, the right hemisphere has a powerful role in human communication and interaction. While the left hemisphere has responsibility for the structure of language (syntax, etc.), the right hemisphere is the domain of the social aspects of communication. The right hemisphere is specialized for attention and is slower and more reflective. The right hemisphere inhibits responses from the left hemisphere that would be problematic, and its role is to combine data to create a perception of the whole. It is responsible for understanding one's relationship with others, including development of empathy and compassion. The right hemisphere comprehends the big picture, while the left hemisphere relishes in detail. The working relationship between the left and right hemispheres becomes problematic when there is a lesion in either hemisphere.

\section{Effects of right hemisphere deficits}

There are five major components of RHD: deficits in motor function, semantic expression and interpretation, pragmatics, speech, and hemispatial awareness. 


\section{Motor deficit}

As with any brain damage, there is the potential for a motor deficit if the areas associated with motor planning or execution are affected. In the case of RHD, lesions may result in hemiparesis if the motor strip is involved, but more often there will be an activation deficit related to hemispatial neglect.

\section{Semantic deficit}

The semantic deficit in RHD is striking in nature, and results in concrete interpretation of meaning by the patient. In normal language processing, the two cerebral hemispheres share semantic interpretation [51]. The frequent result of right hemisphere lesion is that interpretation of word meaning becomes more concrete, with alternate meanings being unexplored by the patient. While concrete interpretation by itself may not be a debilitating problem, individuals with right hemisphere lesions frequently fail to recognize their deficits (as will be discussed), so that failure to identify alternate meanings can result in unnoticed misinterpretations. There are several theories about the root cause of this deficit (e.g., coarse semantic coding $[4,52]$, suppression $[53,54]$ ), but the result is a marked failure in communication. The left hemisphere appears to maintain the denotative or "dictionary meaning" portion of the semantic base, which is the first meaning one thinks of upon hearing a word. The left hemisphere activates this meaning upon being presented with a stimulus, and if this meaning fits the stimulus (e.g., a picture or environmental context), it is kept as the appropriate meaning of the spoken utterance. If, on the other hand, the first interpretation does not align with other information that has been presented (e.g., a mismatch between auditory and visual information or communication context), the right hemisphere is called into action to provide connotative meanings (alternate meanings). Connotative meanings are often words that have emotion attached to them, and they always carry meaning beyond the literal interpretation. The left hemisphere appears to favor the rapidly accessible concrete, denotative meanings that are literal translation of words.

As an example of this dual processing model, a concrete semantic feature map of the word "dog" might include the notion that a dog is a mammal with fur, four legs, ears, mouth, teeth, etc. If someone were to say the sentence "She dogged me until I got the project finished," the left hemisphere would quickly recall the primary definition (four-legged mammal), but the right hemisphere would recognize that this definition did not fit the context of the sentence. The right hemisphere would then be called on to identify alternate meanings for "dog," including "to pester," "to restrain action," and so forth. Based on the context, the right hemisphere can then identify the correct meaning in order to complete the interpretation. A person with RHD might simply state that the sentence is wrong and dismiss it. Thus, the right hemisphere seems to be responsible for identifying the alternate meanings, or at the very least is critical for the process of identifying these alternate meanings. There are many hypothesized reasons for this disparity, and it could simply come down to failure to inhibit response (i.e., lack of reflection [55]).

\section{Humor}

Humor is another very real problem with RHD [56]. Humor, particularly visual humor, depends on an initial mismatch between the image and words, or some other aspect of the image. One looks at an image and the first (left hemisphere) response is the impulsive, rapid interpretation. Continued examination reveals the information that represents the alternate definition or interpretation, and a mismatch between the first and second (perhaps ironic or unexpected) interpretation triggers the humorous reaction through the "perceptual cliff" of a mismatch. A common complaint is that others feel the person with RHD has lost his or her sense of humor.

\section{Idioms}

The right hemisphere has difficulty interpreting literal versus figurative meaning, and thus idioms and metaphors can be a significant problem [57-60]. For example, the idiomatic expression of "having a broken heart" has meaning that falls outside of a concrete interpretation. When presented with an idiom, a person with RHD may simply state that it makes no sense, or alternately provide a meaning that is confabulated to fit the statement.

\section{Cognitive-pragmatic dysfunction}

Cognitive-pragmatic dysfunction is a very large and overarching issue with RHD. People with RHD have difficulty deriving the context to help them plan or organize, because organization requires both right and left DLPFC. Organizing content into categories is particularly difficult because one has to identify common elements among the disparate items. The left hemisphere is very effective at identifying elements, but the commonalities require inference, which is a righthemisphere domain. 
At the core of the pragmatics deficit in RHD is diminished Theory of Mind $[61,62]$. Theory of Mind (TOM) refers to the concept in which an individual develops an internal theory about how another person is thinking, which is a foundational element of interpersonal communication. TOM is largely a right hemisphere function that relies on nuanced information within the communication context $[61,63]$. TOM provides the basis for shared knowledge between two communicators, and allows a communicator to infer the intention of the other member of the communication dyad. When an individual has a right hemisphere lesion, disabling or muting the TOM function can cause difficulty inferring the intention or mental state of another person, subsequently making the person with RHD unaware of how to act.

It is important to recognize that this is not simply that a person is confounded or confused about how to act, but the intrinsic deficit in RHD means that the person may simply not know that there is another way to act. The individual with RHD cannot derive the clues that allow him or her to know what is on the communication partner's mind. Those clues come from the context, facial expression, tone of voice, rate of speech and other domains that a person with RHD simply does not have access to. The right insular cortex is the seat of compassion, and compassion is rooted in the applied, noncynical TOM. While the right insular cortex may not be lesioned itself, it may be inactivated or otherwise inaccessible as a result of the loss of the attentional network.

The pragmatics deficit in RHD leads to inappropriate affect in social situations because the individual cannot recognize the appropriate affect based on cues. The person with RHD fails to read the situation or understand nuance, and the individual's inability to derive the gist of a conversation means that he or she will tend to misunderstand all but the most concrete statements. The person with RHD will give a literal interpretation to idioms or nuanced statements.

With RHD, the person may become an uncomfortable communication partner. Because this individual may have lost sensitivity to pragmatic violations, he or she may not recognize how much knowledge you, the communication partner, have about a topic, or might not see cues signaling that you want to take a turn in the conversation. The person with RHD may not recognize the partner's affect, so the partner may be grieving and the participant with RHD won't recognize it. This is clearly a problem in terms of social interaction. Verbal discourse for people with RHD is also problematic. A person with RHD will have difficulty organizing the discourse, and will not see the relevant cues that might signal topic shift, or that might signal disinterest or interest in a topic. The person with RHD may be very verbose, well beyond the appropriate bounds of conversation $[53,64]$. Similarly, the semantic deficit readily leads to miscommunication based on lack of shared meaning $[65,66]$, causing errors in drawing inferences or interpretation of abstract information.

\section{Speech deficits}

Speech deficits also overlap the pragmatic domain for people with RHD. Because the right hemisphere controls the slower components of speech, fundamental frequency and vocal intensity variation of suprasegmental control may be lost. The speech will likely be more monopitch and monoloud, and the individual will sound disinterested or bored and without affect. The person with RHD may lose the ability to comprehend suprasegmental aspects of speech as well, so that he or she cannot interpret nuance or affect due to the dominance of prosodic processing by the right hemisphere $[67,68]$.

While the deficit in expressing suprasegmental elements of speech causes a flattening of affect for the individual, individuals employing tonal languages such as Mandarin Chinese appear to process the intonation contour within the dominant hemisphere [69] because intonation is intrinsic to semantic meaning in that language (in contrast to right hemisphere processing of intonation in English speakers). Thus, deficits in comprehension and production of prosodic elements may be differentially manifested, depending on the language spoken.

The right hemisphere is also critical to both receptive and expressive emotion processing [70-74]. In addition, individuals experiencing right hemisphere lesions have multi-modal deficits in emotion comprehension, expressed as deficits in processing visual [72] and verbal stimuli [71] for their emotional content, implying a global emotion processing function within this hemisphere.

\section{Hemispatial neglect and misidentification syndromes}

The final key element of RHD is found among what are called misidentification syndromes. Neglect is truly an RHD domain. As we discuss neglect and misidentification syndromes, recognize that there is more at play than simply not being aware of the left side of the body, or the left visual field. The right hemisphere is something of the keeper of the person, or in many sense, the personality. The insular cortex, right claustrum, cuneus, and precuneous are all involved in sense of self and self-agency. Failure to activate the right hemisphere be- 
cause of loss of the executive attention network could result in a core deficit related to affect, motivation, and personality. Fragmentation of the right hemisphere results in fragmentation of that which is the individual. Misidentification syndromes are the heart of the dissociation of the person, with the last of these, hemispatial neglect, being as common in RHD as aphasia is in left-hemisphere dysfunction (LHD).

\section{Misidentification syndromes}

Following is a brief summary of misidentification syndromes that can occur as a part of RHD [75-77].

\section{Asomatognosia}

Asomatognosia refers to unawareness of the ownership of half of the body or of a limb (typically left arm). This is a confusion about ownership, but can be improved through bringing the deficit to the attention of the patient $[78,79]$.

\section{Somatoparaphrenia}

Somatoparaphrenia is also a misidentification of a body part as belonging to someone else, but includes delusions and confabulations about to whom the arm belongs [80]. This person is resistant to any information to the contrary, and will likely not be convinced otherwise. Thus somatoparaphrenia includes denial that prohibits therapeutic correction.

\section{Capgras syndrome}

Capgras syndrome is the belief that a close relative or friend, or even a building has been replaced by an imposter [81]. The individuals might be in their own home and insist that it looks like their home but it really isn't: someone has substituted their home with another home. Similarly, the individual may feel that his or her spouse has been replaced by an imposter. This delusion is considered a psychiatric condition, and is often treated with antipsychotics. It can be found in people who have suffered right hemisphere stroke.

\section{Anosognosia}

Anosognosia refers to a lack of acknowledgement of a disease. This may accompany hemispatial neglect, in that the person doesn't acknowledge that there is paralysis, despite clear evidence [82]. This is a significant barrier to therapeutic progress, as the individual is not aware of a problem despite it having been pointed out to him or her.

Misidentification syndromes cause a loss of connectedness and trust. The person loses familiarity with the environment, and loses trust in others. This person may have increased isolation and paranoia. In the RHD population, the causes of misidentification syndromes seem to revolve around size and location of lesion. Lesions to the right insular cortex, claustrum, precuneus, cuneus, and orbitofrontal cortex reduce the sense of self and self-image. The claustrum coordinates the disparate parts of the cerebrum to orchestrate a wholeness of person, and dysynchrony of the claustrum function could well result in loss of the solid sense of self [83]. Similarly, damage to the temporoparietal juncture and DLPFC are common to all misidentification syndromes, and these structures are intrinsic to the executive attention network. Failure of this network results in an inability to focus attention on relevant stimuli.

Site of lesion for all misidentification syndromes is largely confined to the right hemisphere. Feinberg et al. [84] examined lesion sites along this continuum from paralysis to the profound deficit of somatoparaphrenia (including neglect, delusion, and confabulation) finding that the greater the lesion space, the more significant is the misidentification syndrome. Somatoparaphrenia, with neglect, delusion, and confabulation, has the largest right hemisphere lesion, with paralysis alone being the least. In all cases, the misidentification syndromes share damage to the right DLPFC and TPJ.

\section{Left neglect}

Left neglect, also known as hemispatial neglect, is the dominant syndrome of right hemisphere disorder, and is emblematic of the problems faced by people with RHD. It almost never occurs as a result of damage to the dominant hemisphere, because the attentional network is housed in the nondominant hemisphere.

Barrett [5] identified significant issues related to left neglect that bear on our discussion, as illustrated in Table 2. From a clinical standpoint, one of the most significant of these is loss of awareness of the deficit (anasognosia). The individuals with left neglect may well not be aware that there is a problem and may deny that they have had a stroke. From a therapeutic standpoint this is a daunting challenge, because without awareness of a problem there is no motivation to change. Indeed, the person with right hemisphere cerebrovascular accident (CVA) may not seek medical attention due to loss of awareness of the deficit.

Hypoarousal is a significant contributor to the other issues. The person with left neglect will seem apathetic due to the low state of physiological arousal mediated by the attentional 
Table 2. Common issues associated with left neglect (adapted from Barrett, 2000)

\begin{tabular}{ll}
\hline Lack of awareness & $\begin{array}{c}\text { lack of, or reduced, awareness of the fact that they } \\
\text { are disabled } \\
\text { Denial of deficit (anosognosia) } \\
\text { Unaware of stroke }\end{array}$ \\
Emotion disturbance & $\begin{array}{l}\text { No longer make appropriate facial expressions, flat } \\
\text { intonation } \\
\text { Can't recognize emotions in others } \\
\text { Can't read body language, facial expressions or } \\
\text { emotional content of voice }\end{array}$ \\
Indifferent, apathetic \\
Mypoarousal \\
$\begin{array}{l}\text { Abulia: profound passivity } \\
\text { May make no move to dress selves or explore } \\
\text { environment }\end{array}$ \\
$\begin{array}{l}\text { Difficulty activating motor responses, especially left } \\
\text { limb activation }\end{array}$ \\
Directional hypokinesia: tend not to move left limbs; \\
turn toward right \\
Ask to raise both arms, only raises right arm \\
Neglect left side of own body \\
May even deny ownership of left arm
\end{tabular}

component. This hypoarousal results in profound passivity (abulia), and even reduced motor activation. The individuals may make no effort to dress themselves or to explore the environment. In terms of motor function, the person with left neglect will favor the right hand or leg, with the near total neglect of motor activation of the left side of the body. This neglect will extend to personal hygiene and even to denial of the left side of the body.

Myers [3] has been in the fore-front of RHD for many years, and she has identified a number of components of left neglect (Table 3). These are the classic signs, from grooming and navigating, to neglecting half of a page being read. Individuals with left neglect will very often bump into door frames when walking through a door, will eat only what is on the right side of their plate, and will ignore the left half of an image. Significantly, these individuals will have a great deal of difficulty following the plot of a video, and localizing sounds from the left auditory field. Most importantly, they often do not even realize the depth of the problem. Left neglect extends beyond the neglect of the body, but to the neglect of the problem. It is truly a deficit in the attentional network. The specific site of lesion for left neglect is the right TPJ, and often includes the
Table 3. Signs of left neglect. Adapted from Myers 2010

\begin{tabular}{|c|c|}
\hline Difficulty with & $\begin{array}{l}\text { Responding to individuals or recognizing the presence of } \\
\text { people or objects to the left of their body } \\
\text { Difficulty with self-care on the left side of the body, } \\
\text { including grooming, bathing, eating and dressing } \\
\text { Spontaneous and voluntary movement of limbs on the } \\
\text { left side of the body } \\
\text { Movement through doorways due to bumping into the } \\
\text { wall on the left side } \\
\text { Reading the left half of printed materials } \\
\text { Use of all page space when writing } \\
\text { Getting the gist of presentations or films; following plots } \\
\text { of television shows } \\
\text { Localizing sounds from the left auditory field } \\
\text { Insight or awareness of the physical deficits; includes } \\
\text { awareness of hemiplegia and neglect } \\
\text { Participation in rehabilitation }\end{array}$ \\
\hline
\end{tabular}

right DLPFC.

It is really important to acknowledge the differences between neglect and sensory deficit. In neglect, the patient is ignoring the problem, but in sensory deficit the patient will often complain about the problem. The person with neglect has difficulty with searching, but the patient with a sensory deficit has trouble seeing. Importantly, the patient with neglect will not attempt to compensate, while the person with sensory deficit will [3].

In the motor domain, neglect produces a lack of spontaneous movement in absence of weakness. The clinician will see a failure to adjust posture, and even failure to use the left limb for balance. A person with paresis in absence of neglect will show difficulty exerting force, but will attempt to use the musculature. It will be the limitations of muscular function that cause difficulty.

Reading and writing can be profoundly affected with neglect. As illustrated in Figure 2, the stricken sections are those that the patient with left visual neglect would fail to read when asked to read aloud. This is a very important component of neglect: It is not just that the patient did not read the left side, but the patient very likely would not recognize that the passage didn't make sense. Neglect is not a unitary deficit, but rather is part of a larger failure of the right hemisphere. It appears to arise as a global lack of activation of the right hemisphere through inaction of the attentional network, resulting in a loss of the integrative power of that hemisphere.

In the context of therapy for neglect, increasing the patient's level of arousal will improve his or her ability to scan, thereby 


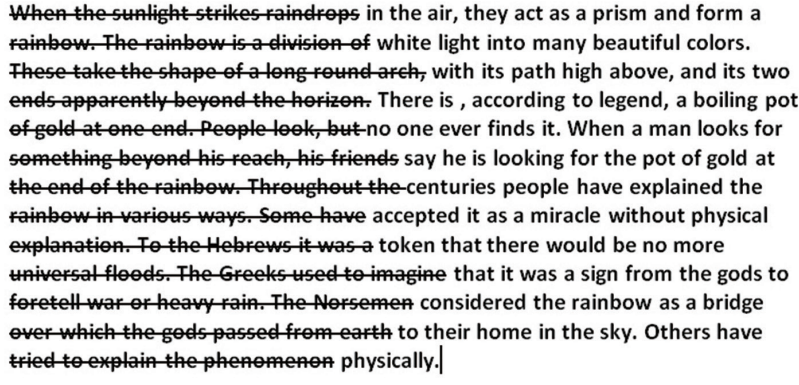

Figure 2. Rainbow passage as perceived by someone with left neglect. Note that words stricken through represent those not read by the patient due to neglect.

decreasing neglect. Some centers are utilizing pharmaceutical means to increase cognitive arousal, and this may be a useful avenue for treatment of neglect syndrome.

\section{Summary}

RHD is a debilitating but seemingly hidden disorder that includes a constellation of subtle but important deficits. Individuals with RHD may demonstrate deficit in a semantic deficit that is characterized by concrete and often erroneous interpretations, failure to recognize errors, and a deficits in pragmatics that manifest as violations of conversational conventions. The individual may also demonstrate hemispatial neglect and/or motor impairment that may extend to denial of the deficit.

The hypoarousal of the right hemisphere results in not only the issues related to hemispatial neglect, but may also may account for loss of affect, caring, empathy and compassion by inactivation of, or lack of access to, the networks associated with Theory of Mind, including the claustrum, right insular cortex, and cuneate and precuneate gyri. That is to say, flattening and diminution of personality in RHD may be a collateral effect of deactivation or lack of access to the essential networks, so that non-lesioned areas are simply not given voice in the face of the very active left hemisphere.

The results of RHD are far-reaching, resulting in loss of ability to interpret humor and idioms, facial expression, verbal intonation, and emotion. The person with RHD may well experience increasing social isolation as the pragmatic deficits alienate others within his or her environment, and the element of denial makes remediation particularly difficult. An important avenue of intervention research should focus on increasing access to the right hemisphere, perhaps by electrophysiological activation. Given increased hemisphere activation the person with RHD might again have normalized ac- cess to the very human characteristics of humor, empathy and compassion and once again experience the richness of life.

\section{REFERENCES}

1. Beeman MJ, Chiarello C. Complementary right-and left-hemisphere language comprehension. Current Directions in Psychological Science. 1998;7(1):2-8.

2. Cai Q, Van der Haegen L, Brysbaert M. Complementary hemispheric specialization for language production and visuospatial attention. Proceedings of the National Academy of Sciences. 2013;110(4):E322-E330.

3. Myers PS. Right hemisphere damage: disorders of communication and cognition. Singular Publishing Group. 1999.

4. Bowden EM, Beeman MJ. Getting the right idea: semantic activation in the right hemisphere may help solve insight problems. Psychological Science. 1998;9(6):435-440.

5. Barrett A. Treatment of unilateral neglect in patients with right hemisphere damage. Neurophysiology and Neurogenic Speech and Language Disorders, Special Interest Group 2 Perspectives on Neurophysiology and Neurogenic Speech and Language Disorders. 2000;12(10):18-26.

6. Vuoksimaa E, Koskenvuo M, Rose RJ, Kaprio J. Origins of handedness: a nationwide study of 30,161 adults. Neuropsychologia. 2009;47(5):1294-1301.

7. Knecht S, Dräger B, Deppe M, Bobe L, Lohmann H, Flöel A, et al. Handedness and hemispheric language dominance in healthy humans. Brain. 2000;123(12):2512-2518.

8. Pulvermüller F. Brain mechanisms linking language and action. Nature Reviews Neuroscience. 2005;6(7):576.

9. Blumenfeld B, Bibitchkov D, Tsodyks M. Neural network model of the primary visual cortex: from functional architecture to lateral connectivity and back. Journal of computational neuroscience. 2006;20(2):219.

10. Wang D, Buckner RL, Liu H. Functional specialization in the human brain estimated by intrinsic hemispheric interaction. Journal of Neuroscience. 2014;34(37):12341-12352.

11. Wey HY, Phillips KA, McKay DR, Laird AR, Kochunov P, Davis MD, et al. Multi-region hemispheric specialization differentiates human from nonhuman primate brain function. Brain Structure and Function. 2014;219(6):2187-2194.

12. Tzourio-Mazoyer N, Perrone-Bertolotti M, Jobard G, Mazoyer B, Baciu M. Multi-factorial modulation of hemispheric specialization and plasticity for language in healthy and pathological conditions: a review. Cortex. 2017;86:314-339.

13. Schwartz J, Tallal P. Rate of acoustic change may underlie hemispheric specialization for speech perception. Science. 1980;207 (4437):1380-1381.

14. Studdert-Kennedy M, Shankweiler D. Hemispheric specialization for speech perception. The Journal of the Acoustical Society of America. 1970;48(2B):579-594.

15. Knoch D, Gianotti LR, Pascual-Leone A, Treyer V, Regard M, 
Hohmann M, et al. Disruption of right prefrontal cortex by lowfrequency repetitive transcranial magnetic stimulation induces risk-taking behavior. Journal of Neuroscience. 2006;26(24):64696472.

16. Blumenfeld RS, Ranganath C. Prefrontal cortex and long-term memory encoding: an integrative review of findings from neuropsychology and neuroimaging. The Neuroscientist. 2007;13(3): 280-291.

17. Lorenz J, Minoshima S, Casey KL. Keeping pain out of mind: the role of the dorsolateral prefrontal cortex in pain modulation. Brain. 2003;126(5):1079-1091.

18. Raz A, Buhle J. Typologies of attentional networks. Nature Reviews Neuroscience. 2006;7(5):367-379.

19. Lochy A, de Heering A, Rossion B. The non-linear development of the right hemispheric specialization for human face perception. Neuropsychologia. 2017.

20. Fridriksson J, Yourganov G, Bonilha L, Basilakos A, Den Ouden D. B, Rorden C. Revealing the dual streams of speech processing. Proceedings of the National Academy of Sciences. 2016;113(52): 15108-15113.

21. Hagoort P, Brown CM, Swaab TY. Lexical-semantic event-related potential effects in patients with left hemisphere lesions and aphasia, and patients with right hemisphere lesions without aphasia. Brain. 1996;119(2):627-649.

22. Hickok G, Poeppel D. The cortical organization of speech processing. Nature Reviews Neuroscience. 2007;8(5):393.

23. Dronkers NF. A new brain region for coordinating speech articulation. Nature. 1996;384(6605):159.

24. Shuren J. Insula and aphasia. Journal of neurology. 1993;240(4): 216-218.

25. Donnan GA, Darby DG, Saling MM. Identification of brain region for coordinating speech articulation. The Lancet. 1997;349(9047): 221-222.

26. Nestor PJ, Graham NL, Fryer TD, Williams GB, Patterson K, Hodges JR. Progressive non-fluent aphasia is associated with hypometabolism centered on the left anterior insula. Brain. 2003;126(11): 2406-2418.

27. Hillis AE, Work M, Barker PB, Jacobs MA, Breese EL, Maurer K. Re-examining the brain regions crucial for orchestrating speech articulation. Brain. 2004;127(7):1479-1487.

28. Moser D, Basilakos A, Fillmore P, Fridriksson J. Brain damage associated with apraxia of speech: evidence from case studies. Neurocase. 2016;22(4):346-356.

29. Craig $\mathrm{AD}$, Craig $\mathrm{AD}$. How do you feel--now? The anterior insula and human awareness. Nature Reviews Neuroscience. 2009;10(1).

30. Brooks JCW, Zambreanu L, Godinez A, Tracey I. Somatotopic organisation of the human insula to painful heat studied with high resolution functional imaging. Neuroimage. 2005;27(1):201-209.

31. Björnsdotter M, Löken L, Olausson H, Vallbo Å, Wessberg J. Somatotopic organization of gentle touch processing in the posterior insular cortex. Journal of Neuroscience. 2009;29(29):9314-9320.

32. Horn NR, Dolan M, Elliott R, Deakin JFW, Woodruff PWR. Response inhibition and impulsivity: an fMRI study. Neuropsycho- logia. 2003;41(14):1959-1966.

33. Miller LA, Collins RL, Kent TA. Language and the modulation of impulsive aggression. The Journal of Neuropsychiatry and Clinical Neurosciences. 2008;20(3):261-273.

34. Bush G, Luu P, Posner MI. Cognitive and emotional influences in anterior cingulate cortex. Trends in Cognitive Sciences. 2000;4(6): 215-222.

35. Etkin A, Egner T, Kalisch R. Emotional processing in anterior cingulate and medial prefrontal cortex. Trends in Cognitive Sciences. 2011;15(2):85-93.

36. Etkin A, Egner T, Peraza DM, Kandel ER, Hirsch J. Resolving emotional conflict: a role for the rostral anterior cingulate cortex in modulating activity in the amygdala. Neuron. 2006;51(6):871-882.

37. Lane RD, Reiman EM, Axelrod B, Yun LS, Holmes A, Schwartz GE. Neural correlates of levels of emotional awareness: evidence of an interaction between emotion and attention in the anterior cingulate cortex. Journal of Cognitive Neuroscience. 1998;10(4):525535.

38. Vogt BA, Finch DM, Olson CR. Functional heterogeneity in cingulate cortex: the anterior executive and posterior evaluative regions. Cerebral Cortex. 1992;2(6):435-443.

39. Beyer C, Anguiano G, Mena F. Oxytocin release in response to stimulation of cingulate gyrus. American Journal of Physiology-Legacy Content. 1961;200(3):625-627.

40. Maddock RJ, Garrett AS, Buonocore MH. Remembering familiar people: the posterior cingulate cortex and autobiographical memory retrieval. Neuroscience. 2001;104(3):667-676.

41. Beauregard M, Levesque J, Bourgouin P. Neural correlates of conscious self-regulation of emotion. The Journal of Neuroscience. 2001.

42. Sandel ME, Mysiw WJ. The agitated brain injured patient. Part 1: Definitions, differential diagnosis, and assessment. Archives of Physical Medicine and Rehabilitation. 1996;77(6):617-623.

43. Bell CC. Coma and the etiology of violence, Part 1. Journal of the National Medical Association. 1986;78(12):1167.

44. Coull JT, Frith CD, Büchel C, Nobre AC. Orienting attention in time: behavioural and neuroanatomical distinction between exogenous and endogenous shifts. Neuropsychologia. 2000;38(6): 808-819.

45. Doniger GM, Foxe JJ, Murray MM, Higgins BA, Snodgrass JG, Schroeder CE, et al. Activation timecourse of ventral visual stream object-recognition areas: high density electrical mapping of perceptual closure processes. Journal of Cognitive Neuroscience. 2000;12(4):615-621.

46. Sammler D, Anwander A, Bestelmeyer PE, Belin P. Dual streams for prosody in the right hemisphere. 2015.

47. Witteman J, Goerlich-Dobre KS, Martens S, Aleman A, Van Heuven VJ, Schiller NO. The nature of hemispheric specialization for prosody perception. Cognitive, Affective, \& Behavioral Neuroscience. 2014;14(3):1104-1114.

48. Costanzo EY, Villarreal M, Drucaroff LJ, Ortiz-Villafane M, Castro $\mathrm{MN}$, Goldschmidt M, et al. Hemispheric specialization in affective responses, cerebral dominance for language, and handedness: 
lateralization of emotion, language, and dexterity. Behavioural Brain Research. 2015;288:11-19.

49. Dimberg U, Petterson M. Facial reactions to happy and angry facial expressions: evidence for right hemisphere dominance. Psychophysiology. 2000;37(5):693-696.

50. Youse KM, Coelho CA. Treating underlying attention deficits as a means for improving conversational discourse in individuals with closed head injury: a preliminary study. Neurorehabilitation. 2009;24(4):355-364.

51. Pobric G, Lambon Ralph MA, Zahn R. Hemispheric specialization within the superior anterior temporal cortex for social and nonsocial concepts. Journal of Cognitive Neuroscience. 2016;28(3):351360 .

52. Tompkins CA, Scharp VL, Meigh KM, Fassbinder W. Coarse coding and discourse comprehension in adults with right hemisphere brain damage. Aphasiology. 2008;22(2):204-223.

53. Tompkins CA, Baumgaertner A, Lehman MT, Fassbinder W. Mechanisms of discourse comprehension impairment after right hemisphere brain damage: suppression in lexical ambiguity resolution. Journal of Speech, Language, and Hearing Research. 2000;43(1):62-78.

54. Tompkins CA, Blake MT, L Scharp V, Meigh KM, Wambaugh JL. Implicit treatment of underlying comprehension processes improves narrative comprehension in right hemisphere brain damage. 2013.

55. Tompkins CA, Lehman-Blake MT, Baumgaertner A, Fassbinder W. Mechanisms of discourse comprehension impairment after right hemisphere brain damage: suppression in inferential ambiguity resolution. Journal of Speech, Language, and Hearing Research. 2001;44(2):400-415.

56. Heath RL, Blonder LX. Spontaneous humor among right hemisphere stroke survivors. Brain and Language. 2005;93(3):267-276.

57. Lundgren K, Brownell H, Cayer-Meade C, Milione J, Kearns K. Treating metaphor interpretation deficits subsequent to right hemisphere brain damage: preliminary results. Aphasiology. 2011;25(4):456-474.

58. Myers PS, Linebaugh CW. Comprehension of idiomatic expressions by right-hemisphere-damaged adults. In Clinical Aphasiology: Proceedings of the Conference 1981 (p. 254-261) BRK Publishers. 1981.

59. Papagno C, Curti R, Rizzo S, Crippa F, Colombo MR. Is the right hemisphere involved in idiom comprehension? A neuropsychological study. Neuropsychology. 2006;20(5):598.

60. Van Lancker DR, Kempler D. Comprehension of familiar phrases by left-but not by right-hemisphere damaged patients. Brain and Language. 1987;32(2):265-277.

61. Kalbe E, Schlegel M, Sack AT, Nowak DA, Dafotakis M, Bangard C, et al. Dissociating cognitive from affective theory of mind: a TMS study. Cortex. 2010;46(6):769-780.

62. Weed E, McGregor W, Nielsen JF, Roepstorff A, Frith U. Theory of Mind in adults with right hemisphere damage: what's the story? Brain and Language. 2010;113(2):65-72.

63. Viggiano MP, Giovannelli F, Borgheresi A, Feurra M, Berardi N,
Pizzorusso T, et al. Disruption of the prefrontal cortex function by rTMS produces a category-specific enhancement of the reaction times during visual object identification. Neuropsychologia. 2008;46(11):2725-2731.

64. Blake ML. Clinical relevance of discourse characteristics after right hemisphere brain damage. American Journal of SpeechLanguage Pathology. 2006;15(3):255-267.

65. Beeman M. Semantic processing in the right hemisphere may contribute to drawing inferences from discourse. Brain and Language. 1993;44(1):80-120.

66. Brownell HH, Simpson TL, Bihrle AM, Potter HH, Gardner H. Appreciation of metaphoric alternative word meanings by left and right brain-damaged patients. Neuropsychologia. 1990;28(4):375383.

67. Cohen MJ, Branch WB, Hynd GW. Receptive prosody in children with left or right hemisphere dysfunction. Brain and Language. 1994;47(2):171-181.

68. Yang SY, Sidtis DVL. Production of Korean idiomatic utterances following left-and right-hemisphere damage: acoustic studies. Journal of Speech, Language, and Hearing Research. 2016;59(2): 267-280.

69. Luo H, Ni JT, Li ZH, Li XO, Zhang DR, Zeng FG, et al. Opposite patterns of hemisphere dominance for early auditory processing of lexical tones and consonants. Proceedings of the National Academy of Sciences. 2006;103(51):19558-19563.

70. Borod JC, Andelman F, Obler LK, Tweedy JR, Wilkowitz J. Right hemisphere specialization for the identification of emotional words and sentences: evidence from stroke patients. Neuropsychologia. 1992;30(9):827-844.

71. Borod JC, Cicero BA, Obler LK, Welkowitz J, Erhan HM, Santschi C, et al. Right hemisphere emotional perception: evidence across multiple channels. Neuropsychology. 1998;12(3):446.

72. Blonder LX, Bowers D, Heilman KM. The role of the right hemisphere in emotional communication. Brain. 1991;114(3):1115-1127.

73. George MS, Parekh PI, Rosinsky N, Ketter TA, Kimbrell TA, Heilman KM, et al. Understanding emotional prosody activates right hemisphere regions. Archives of Neurology. 1996;53(7):665-670.

74. Schmidt AT, Hanten GR, Li X, Orsten KD, Levin HS. Emotion recognition following pediatric traumatic brain injury: longitudinal analysis of emotional prosody and facial emotion recognition. Neuropsychologia. 2010;48(10):2869-2877.

75. Feinberg TE. Neuropathologies of the self: clinical and anatomical features. Consciousness and Cognition. 2011;20(1):75-81.

76. Feinberg TE. Neuropathologies of the self and the right hemisphere: a window into productive personal pathologies. Frontiers in Human Neuroscience. 2013;7.

77. Feinberg TE, Venneri A, Simone AM, Fan Y, Northoff G. The neuroanatomy of asomatognosia and somatoparaphrenia. Journal of Neurology, Neurosurgery \& Psychiatry. 2010;81(3):276-281.

78. Arzy S, Overney LS, Landis T, Blanke O. Neural mechanisms of embodiment: asomatognosia due to premotor cortex damage. Archives of Neurology. 2006;63(7):1022-1025.

79. Blom RM, Hennekam RC, Denys D. Body integrity identity disor- 
der. PLoS One. 2012;7(4):e34702.

80. Vallar G, Ronchi R. Somatoparaphrenia: a body delusion. A review of the neuropsychological literature. Experimental Brain Research. 2009;192(3):533-551.

81. Ferguson MR, Yu CK, Poliakov AV, Friedman SD, McClellan JM. Capgras syndrome: neuroanatomical assessment of brain MRI findings in an adolescent patient. Brain. 2017;140(7):e43.

82. Vannini P, Hanseeuw B, Munro CE, Amariglio RE, Marshall GA, Rentz DM, et al. Anosognosia for memory deficits in mild cogni- tive impairment: insight into the neural mechanism using functional and molecular imaging. NeuroImage: Clinical. 2017;15, 408-414.

83. Crick FC, Koch C. What is the function of the claustrum? Philosophical Transactions of the Royal Society B. Biological Sciences. 2005;360(1458):1271-1279.

84. Feinberg TE, Roane DM. Delusional misidentification. Psychiatric Clinics. 2005;28(3):665-683. 